\title{
Effect of Seedling Age and Weed Management on Growth and Yield of Transplanted Rice (Sakha $106 \mathrm{cv}$ ).
}

\author{
El-Ghandor ${ }^{1}$, A. M. A. and A. M. Khozimy ${ }^{2}$ \\ ${ }^{1}$ Rice Research Department, Field Crops Research Institute, ARC, Giza, Egypt \\ ${ }^{2}$ Plant Protection Department, Faculty of Agriculture, Damanhour University, Egypt
}

Received: $21 / 9 / 2019$

\begin{abstract}
Two field experiments were conducted at the Experimental Farm of Sakha Agricultural Research Station, Kafrelsheikh, Egypt during 2016 and 2017 summer seasons to study the effect of seedling age and weed management on growth and yield of transplanted rice (Sakha $106 \mathrm{cv}$ ). A split plot experimental design arranged in randomized complete block with four replications was used in both seasons. Three seedling ages viz., 21, 28 and 35 days were allocated to the main plots, while five weed control treatments i.e., thiobencarb $(50 \% \mathrm{EC})$ at $2.39 \mathrm{~kg} \mathrm{ai} \mathrm{ha}^{-1}$, oxadiazon $\left(25 \%\right.$ EC) at $0.045 \mathrm{~kg}$ ai ha ${ }^{-1}$, penoxsulam $(24 \% \mathrm{SC})$ at $0.029 \mathrm{~kg}^{-a i} \mathrm{ha}^{-1}$, hand weeding two times $(20$ and $40 \mathrm{DAT})$ and untreated (weedy check) were assigned to the sub-plots. The results showed transplanting rice seedlings at 21 days old recorded the lowest dry weight of Eclipta sp C. difformis E crus-galli and total weeds. Also, the increasing in rice dry weight, number of panicles $\mathrm{m}^{-2}$, panicle weight, number of filled grains panicle $\mathrm{e}^{-1}, 1000$-grain weight and rice grain yield as compared to rest seedling ages (28 and 35 days). The application of penoxsulam at $0.029 \mathrm{~kg}^{2}$ ai ha ${ }^{-1}$ applied at 15 DAT achieved the lowest dry weight of weed species and total weeds as well as the highest rice yield as compared to weedy check. The interaction between seedling ages and weed control treatments had significant effect on dry weights of weed species, total weeds dry, rice yield and its components. Where, transplanting rice seedlings treatment at 21 days old treated with herbicide of penoxsulam at $0.029 \mathrm{~kg}$ ai ha ${ }^{-1}$ at $15 \mathrm{DAT}$ are the best among the studied treatments to achieve the best weed control and the highest rice dry mater, grain yield and its components for Sakha $106 \mathrm{cv}$. under this study conditions.
\end{abstract}

Keywords: Seedling age, Weed control, Penoxsulam, Total weeds, Rice grain yield

\section{INTRODUCTION}

Rice (Oryza sativa L.) is considered one of the important cereal crops not only in Egypt but also in all over the world and the main food for more than half of the world population. FAOSTAT (2017) stated that the Egyptian harvested area of about $1,632,461$ million feddan with production of about of 6.38 million tonnes and average productivity of 3.908 tonnes per feddan. Increasing rice grain yield per unit area is considered a local goal to meet the growing demand of this crop. Among various factors affecting rice production, such as seedling age, planting methods, cultivars, transplanting space (El-Kassaby et al., 2012), controlling weeds remain the most important agronomic practice.

Seedling age at the time of transplanting is an important factor in rice production and regulating its growth as it primarily contributes to increase number of productive tillers, panicle length, filled grains panicle ${ }^{-1}$ and 1000-grain weight, ultimately resulted in increased grain yield of rice (Ginigaddara and Ranamukhaarachchi, 2011; El-Khoby and Alaa, 2016). In case of the age of seedlings is increased than optimum, the seedlings produce less number of tillers and dry weight due to lack period of vegetative growth resulting in reduction rice grain yield (Roy, 2015).

Kewat et al. (2002) and Krishna et al. (2008) reported that young seedling at age of 21 days old produced higher number of tillers, which contributed to higher grain yield more than 28 days old seedlings. Abou-Khalifa (2005) reported that seedling age of 25 days produced the highest value of number of panicles $\mathrm{m}^{-2}, 1000$-grain weight, panicle weight, number of filled grains panicle ${ }^{-1}$ and grain yield. Upandhyay (2003) and
Salem et al. (2011) found that the youngest seedling (20 days old) recorded the highest dry weight, number of tillers $\mathrm{m}^{-2}, 1000$-grain weight, number of grain panicle ${ }^{-1}$ and grain yield more than 30 and 40 day old seedlings.

Weeds are one of the most important serious resources considered in rice production. Weeds compete with crops on water, nutrients, light and space. Moreover, it is the important competitor in their early growth stages resulting in reduced the growth of crops and finally grain yield (Shukla et al. 2014). Losses caused by weeds vary from one country to another, depending on weed species prevailing, planting method common and the control methods practiced by farmers. These losses were estimated at 36-90\% in Egypt (Hassan, 1999), 40\% in Bangladesh (Karim et al, 1998) and $10-85 \%$, in general, (Labrada, 2001). Rice yield losses due to weed competition vary, depending on method of planting. The losses ranged from $14-93 \%$ in direct-seeded rice, $17-47 \%$ in transplanted rice (Ranjit, 1997), and ranged from 40-80\% in direct-sown rain fed upland rice (Thakur and Bassi, 1994).

The most problematic weeds in rice field are grasses (Echinochloa crus galli and Echinochloa colona) and sedges (Cyperus rotundus, Cyperus difformis and Cyperus irria) (Sandeep et al., 2002, Rekha et al., 2003 and Ahmad et al., 2004). These weeds compete with rice plant on nutrition, light, space and air. For this reason produces lose in rice yields.

The traditional methods of weed control in rice include hand weeding; it is easy method and environment-friendly but this is becoming less common because of labour scarcity at critical time of weeding and increasing labour costs (Kumar and Ladha, 2011; Chauhan, 2012). Moreover, morphological similarity 
between grassy weeds and rice seedlings makes hand weeding difficult at early stages of growth (Rahman et al., 2012). Nowadays, herbicides are becoming the alternative to weed control in rice culture due to their rapid effects and it is more effective, easy to apply, economically available method for controlling different weed species at the same time and less cost compared to traditional methods weeding (Singh et al., 2016). Therefore, the objectives of this study were to evaluate the best seedling age, to determine the best weed control method and to evaluate the interaction effects between seedling age and weed control methods on growth and rice grain yield (Sakha106 cv).

\section{MATERIALS AND METHODS}

Two field experiments were conducted in 2016 and 2017 seasons at the Experimental Farm of Sakha, Agricultural Research Station, ARC, Egypt to study the effect of seedling age and weed management on growth, yield and its attributes of Sakha 106 rice cultivar under transplanting method. A split plot experimental design arranged in randomized complete block with four replications was used in both seasons. Main plots were devoted to three seedling ages while, weed control treatments were distributed with sub-plots in plot area of $20 \mathrm{~m}^{2}(4 \times 5 \mathrm{~m})$. Rice cultivar was transplanted on $3^{\text {rd }}$ and $5^{\text {th }}$ of May in 2016 and 2017 seasons, respectively. Rice seeds were soaked in fresh water for 48 hours and then incubated for another 48 hour to enhance germination. Pre-germinated seeds were broadcasted in the presence of water after puddling the nursery. Rice was transplanted at 3-4 seedlings per hill. All cultural practices; i.e., land preparation, phosphorus fertilization and pest management were done as recommended in transplanted rice.

\section{Studied factors were as follow:}

\section{A- Seedling age:}

Three seedling ages were studies as follows:

1- Twenty one days old (Three weeks)

2- Twenty eight days old (four weeks)

3- Thirty five days old (five weeks)

\section{B - Weed control treatments:}

Five weed control treatments were used during the two seasons as follows:

1- Thiobencarb $50 \%$ EC (Saturn) at the rate of 2.39 $\mathrm{kg}$ ai ha ${ }^{-1}$

2- Oxadiazon $25 \%$ EC (Ronstar) at the rate of $0.045 \mathrm{~kg}$ ai ha ${ }^{-1}$

3- Penoxsulam 24\% SC (Granite) at the rate of $0.029 \mathrm{~kg}$ ai ha ${ }^{-1}$

4- Hand weeding two times

5- Untreated (weedy check)

Thiobencarb 50\% EC and oxadiazon $25 \%$ EC as pre-emergence herbicides were applied at 4 days after transplanting (DAT) while, herbicide was deluted by water and mixed with sand, then broadcasted by hand on to flooded plots then, kept field flooded from 3 to 4 days after herbicidal application. Penoxsulam 24\% SC was applied at 15 DAT and it was sprayed in 300 liter water per hectare on wet land by using knapsack sprayer then the soil was flush irrigated after 24 hours from herbicidal application. Hand weeding was applied two times at 20 and 40 DAT.

At 50 DAT, weed samples were collected using $0.5 \mathrm{~m}^{2}$ quadrate $(50 \times 50 \mathrm{~cm})$ replicated four times for each plots. Weeds were cleaned and classified according to it species. The weed plants were obtained and weighed as fresh weight then air dried, then oven dried at $70{ }^{\circ} \mathrm{C}$ up to content weights, the average weight was recorded as $\mathrm{gm}^{-2}$.

Weed control efficiency (WCE \%): WCE was calculated by using the following formula (Drost and Moody, 1982) :

$$
\operatorname{WCE}(\%)=\frac{\text { DMC - DMT }}{-----100}
$$

Where: $\mathbf{D M C}=$ Weed dry matter in weedy check plots

$$
\text { DMT }=\text { Weed dry matter in weed control treatment }
$$

For rice, dry weight was measured at the same time of sampling by the same method (50 DAT). Before harvest, panicles were counted in two random quadrates of $50 \times 50 \mathrm{~cm}$ and number of panicles per square meter was recorded. After rice maturity, panicle weight $(\mathrm{g})$ was estimated by weighing ten random panicles per plot and their average was estimated. Thousand-grain weight (g) was recorded in random samples from grain yield. Number of field grain panicle ${ }^{-1}$ was estimated at ten matured random panicles was recorded. The central 5 $\mathrm{m}^{2}$ from each plot were manually harvested, air dried, threshed and cleaned to determine grain yield $\mathrm{ha}^{-1}$ then rice grain yield was recorded at $14 \%$ moisture content.

\section{Statistical analysis:}

Data analysis: the collected data were subjected to proper statistical analysis of variance according to Snedecor and Cochran (1971). Weed data were statistically analyzed by MSTATC program after transformed according to square-root transformation $(\sqrt{ }[x+0.5])$, while rice collected data were directly analyzed by MSTATC program then the means of both weeds and rice characters were compared by using Duncan's Multiple Range Test (Duncan, 1955).

\section{RESULTS AND DISCUSSION}

\section{a. Weeds:}

The common weed species in the experimental field associated with rice crop during the two growing seasons were: Among the broad leaved weeds was Eclipta sp., the major sedge was Cyperus difformis (small flower) and among the grasses was Echinochloa crus- galli (barnyard grass).

a.1. Effect of seedling age and weed control treatments on dry weight of Eclipta sp, C. difformis, $E$. crus-galli, total weeds and weed control efficiency (\%) during 2016 and 2017 seasons:

Dry weight of Eclipta sp, C. difformis, E. crusgalli, total weeds and weed control efficiency were significantly affected by seedling age and weed control treatments in 2016 and 2017 seasons are presented in Table (1). 
Data indicated that transplanting rice seedlings at 21 day age recorded less dry weight of weed species and total weeds followed by 28 days old as compared to planting at 35 days old which recorded greater dry weight of these traits during two seasons of study. The reduction in dry weight of weeds may be due to the ability of young seedlings ( 21 days old) to deplete more nutrients from soil and, suppress weeds due to enough coverage of leaves and produce higher number of tillers through early growth stage more than number of total weeds, resulting reduced growth and population of weed. The obtained results are confirmed with those obtained by Kavitha et al. (2010), Anitha and Chellappan (2011) and Shukla et al. (2015).

In respect to weed control treatments, all weed control treatments significantly reduced dry weight of
Eclipta sp, C. difformis, E. crus-galli and total weeds as compared to weedy check plots during two seasons of study. The application of post-emergence herbicide ( Penoxsulam 24\% SC ) at the recommended dose ( 0.029 $\mathrm{kg}$ ai ha ${ }^{-1}$ ) recorded the lowest values of weeds dry weight and total weeds while, the highest values of weed species dry and total weeds weight were found in weedy check plots in 2016 and 2017 seasons. The significant reduction in weed characters may refer to high efficiency of chemical weed control treatment, which suppressed weed germination and kill weeds in early stage. These findings are in harmony with these reported by Shebl et al. (2009), Ramachandra et al. (2012) and Mondal et al. (2013).

Table (1): Dry weight (g) of Eclipta sp., C. difformis, E. crus-galli, total weeds and weed control efficiency (\%) as affected by seedling age and weed control treatments in 2016 and 2017 seasons

\begin{tabular}{|c|c|c|c|c|c|c|c|c|c|c|}
\hline \multirow{2}{*}{$\begin{array}{l}\text { Treatments } \\
\text { a- Seedling age }\end{array}$} & \multicolumn{2}{|c|}{ Eclipta sp. } & \multicolumn{2}{|c|}{ C. difformis } & \multicolumn{2}{|c|}{ E. crus-galli } & \multicolumn{2}{|c|}{ Total weeds } & \multicolumn{2}{|c|}{$\begin{array}{l}\text { Weed control } \\
\text { Efficiency }(\%)\end{array}$} \\
\hline & 2016 & 2017 & 2016 & 2017 & 2016 & 2017 & 2016 & 2017 & 2016 & 2017 \\
\hline 21 day & $\begin{array}{c}9.6 \\
(2.5 \mathrm{c})\end{array}$ & $\begin{array}{c}7.7 \\
(2.2 \mathrm{c})\end{array}$ & $\begin{array}{c}31.2 \\
(3.9 \mathrm{c})\end{array}$ & $\begin{array}{c}28.7 \\
(3.6 \mathrm{c})\end{array}$ & $\begin{array}{c}54.9 \\
(4.8 \mathrm{c})\end{array}$ & $\begin{array}{c}52.7 \\
(4.6 \mathrm{c})\end{array}$ & $\begin{array}{c}95.7 \\
(6.6 \mathrm{c})\end{array}$ & $\begin{array}{c}89.0 \\
(6.2 \mathrm{c})\end{array}$ & -- & -- \\
\hline 28 day & $\begin{array}{c}14.6 \\
(3.2 \mathrm{~b})\end{array}$ & $\begin{array}{l}12.3 \\
(2.8 \mathrm{~b})\end{array}$ & $\begin{array}{c}39.1 \\
(4.8 \mathrm{~b})\end{array}$ & $\begin{array}{c}35.7 \\
(4.4 \mathrm{~b})\end{array}$ & $\begin{array}{c}77.7 \\
(5.7 \mathrm{~b})\end{array}$ & $\begin{array}{c}68.7 \\
(5.2 \mathrm{~b})\end{array}$ & $\begin{array}{c}131.4 \\
(8.1 \mathrm{~b})\end{array}$ & $\begin{array}{l}116.7 \\
(7.3 \mathrm{~b})\end{array}$ & -- & -- \\
\hline 35 day & $\begin{array}{c}27.8 \\
(4.7 \mathrm{a})\end{array}$ & $\begin{array}{c}23.5 \\
(4.3 \mathrm{a})\end{array}$ & $\begin{array}{c}70.0 \\
(5.9 \mathrm{a})\end{array}$ & $\begin{array}{c}63.4 \\
(5.4 \mathrm{a})\end{array}$ & $\begin{array}{l}134.2 \\
(9.3 \mathrm{a})\end{array}$ & $\begin{array}{c}127.6 \\
(9.0 \mathrm{a})\end{array}$ & $\begin{array}{c}231.9 \\
(12.1 \mathrm{a})\end{array}$ & $\begin{array}{c}214.5 \\
(11.5 a)\end{array}$ & -- & -- \\
\hline F. test & $* *$ & $* *$ & $* *$ & $* *$ & $* *$ & $* *$ & $* *$ & $* *$ & -- & -- \\
\hline \multicolumn{11}{|l|}{ b- Weed control } \\
\hline Thiobencarb 50\% EC & $\begin{array}{c}14.1 \\
(3.6 \mathrm{~b})\end{array}$ & $\begin{array}{c}10.5 \\
(3.2 \mathrm{~b})\end{array}$ & $\begin{array}{c}25.1 \\
(5.0 \mathrm{~b})\end{array}$ & $\begin{array}{c}17.7 \\
(4.2 \mathrm{~b})\end{array}$ & $\begin{array}{c}44.2 \\
(6.1 \mathrm{~b})\end{array}$ & $\begin{array}{c}40.8 \\
(5.7 \mathrm{~b})\end{array}$ & $\begin{array}{c}83.5 \\
(8.7 \mathrm{~b})\end{array}$ & $\begin{array}{c}69.1 \\
(7.9 \mathrm{~b})\end{array}$ & 64.78 & 66.95 \\
\hline Oxadiazon $25 \%$ EC & $\begin{array}{c}6.7 \\
(2.3 \mathrm{~d})\end{array}$ & $\begin{array}{c}5.5 \\
(2.2 \mathrm{~d})\end{array}$ & $\begin{array}{c}3.9 \\
(1.9 \mathrm{~d})\end{array}$ & $\begin{array}{c}1.8 \\
(1.5 \mathrm{~d})\end{array}$ & $\begin{array}{c}13.7 \\
(3.0 \mathrm{~d})\end{array}$ & $\begin{array}{c}12.6 \\
(2.8 \mathrm{~d})\end{array}$ & $\begin{array}{c}24.2 \\
(4.3 \mathrm{~d})\end{array}$ & $\begin{array}{c}19.9 \\
(3.8 \mathrm{~d})\end{array}$ & 82.59 & 84.10 \\
\hline Penoxsulam 24\% SC & $\begin{array}{c}0.8 \\
(1.0 \mathrm{e})\end{array}$ & $\begin{array}{c}0.4 \\
(0.9 \mathrm{e})\end{array}$ & $\begin{array}{c}0.7 \\
(1.0 \mathrm{e})\end{array}$ & $\begin{array}{c}0.4 \\
(0.9 \mathrm{e})\end{array}$ & $\begin{array}{c}0.7 \\
(1.0 \mathrm{e})\end{array}$ & $\begin{array}{c}0.3 \\
(0.9 \mathrm{e})\end{array}$ & $\begin{array}{c}2.1 \\
(1.5 \mathrm{e})\end{array}$ & $\begin{array}{c}1.1 \\
(1.2 \mathrm{e})\end{array}$ & 93.93 & 94.98 \\
\hline Hand weeding & $\begin{array}{c}8.5 \\
(2.8 \mathrm{c})\end{array}$ & $\begin{array}{c}6.9 \\
(2.5 \mathrm{c})\end{array}$ & $\begin{array}{c}6.9 \\
(2.7 \mathrm{c})\end{array}$ & $\begin{array}{c}5.1 \\
(2.3 \mathrm{c})\end{array}$ & $\begin{array}{c}17.7 \\
(3.8 \mathrm{c})\end{array}$ & $\begin{array}{c}16.1 \\
(3.6 \mathrm{c})\end{array}$ & $\begin{array}{c}33.2 \\
(5.4 \mathrm{c})\end{array}$ & $\begin{array}{c}28.0 \\
(4.9 \mathrm{c})\end{array}$ & 78.14 & 79.50 \\
\hline Weedy check & $\begin{array}{c}56.5 \\
(7.5 \mathrm{a})\end{array}$ & $\begin{array}{c}49.1 \\
(6.9 \mathrm{a})\end{array}$ & $\begin{array}{c}197.2 \\
(13.8 \mathrm{a})\end{array}$ & $\begin{array}{c}188.2 \\
(13.5 a)\end{array}$ & $\begin{array}{c}368.4 \\
(19.0 \mathrm{a})\end{array}$ & $\begin{array}{c}344.9 \\
(18.4 a)\end{array}$ & $\begin{array}{c}622.0 \\
(24.7 a)\end{array}$ & $\begin{array}{c}582.2 \\
(23.9 a)\end{array}$ & 0.00 & 0.00 \\
\hline F. test & $* *$ & $* *$ & $* *$ & $* *$ & $* *$ & $* *$ & $* *$ & $* *$ & -- & -- \\
\hline $\begin{array}{l}\text { Interaction } \\
(\mathrm{a} * \mathrm{~b})\end{array}$ & $* *$ & $* *$ & $* *$ & $* *$ & $* *$ & $* *$ & $* *$ & $* *$ & -- & -- \\
\hline
\end{tabular}

As shown in Table (1), weed control treatments had a significant effect on weed control efficiency percentages $(\%)$. The highest weed control efficiency (\%) was observed in plots treated with penoxsulam $24 \% \mathrm{SC}$ at the rate of $0.029 \mathrm{~kg}$ ai $\mathrm{ha}^{-1}$ followed by oxadiazon $25 \%$ EC at the rate of $0.045 \mathrm{~kg}$ ai ha ${ }^{-1}$. The reason in this might be due to the high efficiency of herbicide in weed control and the reduction in weed germination and growth. These results are confirmed with those cited by Paramita et al. (2005) and Montazeri et al. (2010). On the other hand, the lower weed control efficiency (\%) was observed in weedy check plots during two seasons of study.

a.2. Effect of interaction between seedling ages and weed control treatments on dry weight of Eclipta, $C$. difformis, E. crus-galli and total weeds during 2016 and 2017 seasons:

As shown in Table (2) generally, all weed control treatments caused significant reduction in dry weight of Eclipta, C. difformis, E. crus-galli and total weeds under different seedling ages during two seasons. 


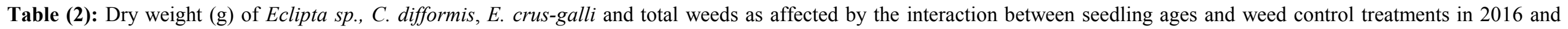
2017 seasons

\begin{tabular}{|c|c|c|c|c|c|c|c|c|c|c|c|c|}
\hline \multirow{4}{*}{$\begin{array}{l}\text { Weed control } \\
\text { treatments }\end{array}$} & \multicolumn{2}{|c|}{ Eclipta sp. } & \multicolumn{3}{|c|}{ C. difformis } & \multicolumn{3}{|c|}{ E. crus-galli } & \multicolumn{4}{|c|}{ Total weeds } \\
\hline & \multicolumn{12}{|c|}{ Seedling age } \\
\hline & 21 days & 28 days & 35 days & 21 days & 28 days & 35 days & 21 days & 28 days & 35 days & 21 days & 28 days & 35 days \\
\hline & \multicolumn{12}{|c|}{2016 Season } \\
\hline Thiobencarb 50\% & 13.1 & 29.0 & 33.4 & 3.9 & 16.7 & 21.7 & 15.9 & 16.5 & 100.2 & 32.9 & 62.3 & 155.2 \\
\hline & $(2.06 \mathrm{fg})$ & $(4.14 \mathrm{e})$ & $(4.71 \mathrm{~d})$ & $(3.66 \mathrm{~d})$ & $(5.43 \mathrm{c})$ & $(5.81 \mathrm{c})$ & $(4.01 \mathrm{f})$ & $(4.13 \mathrm{f})$ & $(10.03 \mathrm{~d})$ & $(5.77 \mathrm{~g})$ & (7.92 ef) & $(12.48 \mathrm{~d})$ \\
\hline Oxadiazon $\quad 25 \%$ & 1.8 & 6.3 & 3.6 & 1.7 & 1.9 & 16.5 & 0.8 & 1.9 & 38.3 & 4.3 & 10.1 & 58.3 \\
\hline & $(1.41 \mathrm{~h})$ & $(1.48 \mathrm{~h})$ & $(4.12 \mathrm{e})$ & $(1.44 \mathrm{fg})$ & $(2.55 \mathrm{e})$ & $(1.85 \mathrm{f})$ & (1.10 hi) & $(1.54 \mathrm{~h})$ & $(6.23 \mathrm{e})$ & $(2.13 \mathrm{j})$ & $(3.20 \mathrm{i})$ & $(7.66 \mathrm{f})$ \\
\hline Penoxsulam $24 \%$ & 0.0 & 0.5 & 1.6 & 0.0 & 0.1 & 2.2 & 0.0 & 0.5 & 1.5 & 0.0 & 1.2 & 5.3 \\
\hline SC & $(0.71 \mathrm{i})$ & $(0.77 \mathrm{i})$ & $(1.64 \mathrm{gh})$ & $(0.71 \mathrm{~h})$ & $(0.99 \mathrm{gh})$ & $(1.43 \mathrm{fg})$ & (0.71 i) & (0.99 hi) & $(1.34 \mathrm{~h})$ & $(0.711)$ & $(1.28 \mathrm{k})$ & $(2.39 \mathrm{j})$ \\
\hline \multirow{2}{*}{ Hand weeding } & 3.0 & 7.9 & 10.0 & 3.3 & 5.0 & 17.2 & 4.7 & 5.8 & 42.7 & 10.9 & 18.8 & 69.9 \\
\hline & $(1.95 \mathrm{fg})$ & $(2.35 \mathrm{f})$ & $(4.20 \mathrm{e})$ & $(1.85 \mathrm{f})$ & $(2.89 \mathrm{e})$ & $(3.22 \mathrm{de})$ & $(2.27 \mathrm{~g})$ & $(2.51 \mathrm{~g})$ & $(6.57 \mathrm{e})$ & (3.38 i) & $(4.38 \mathrm{~h})$ & $(8.39 \mathrm{e})$ \\
\hline \multirow[t]{2}{*}{ Weedy check } & 137.9 & 152.0 & 301.6 & 39.0 & 49.3 & 81.2 & 253.3 & 363.7 & 488.2 & 430.2 & 564.9 & 871.0 \\
\hline & $(6.29 \mathrm{c})$ & $(7.05 \mathrm{~b})$ & $(9.04 \mathrm{a})$ & $(11.76 \mathrm{~b})$ & $(12.34 \mathrm{~b})$ & $(17.38 \mathrm{a})$ & $(15.93 \mathrm{c})$ & $(19.08 \mathrm{~b})$ & $(22.11 \mathrm{a})$ & $(20.75 \mathrm{c})$ & $(23.77 \mathrm{~b})$ & $(29.52 \mathrm{a})$ \\
\hline \multicolumn{13}{|c|}{2017 Season } \\
\hline Thiobencarb 50\% & 9.8 & 20.4 & 22.9 & 3.0 & 13.2 & 15.4 & 13.3 & 13.8 & 95.5 & 26.1 & 47.3 & 133.8 \\
\hline EC & $(1.84 \mathrm{e})$ & $(3.66 \mathrm{~d})$ & $(3.99 \mathrm{~d})$ & $(3.19 \mathrm{e})$ & $(4.57 \mathrm{~d})$ & $(4.83 \mathrm{~d})$ & $(3.66 \mathrm{f})$ & $(3.77 \mathrm{f})$ & $(9.79 \mathrm{~d})$ & $(5.13 \mathrm{~g})$ & $(6.90 \mathrm{f})$ & $(11.58 \mathrm{~d})$ \\
\hline Oxadiazon & 1.1 & 2.6 & 1.6 & 1.2 & 1.6 & 13.8 & 0.6 & 1.0 & 36.1 & 2.9 & 5.3 & 51.5 \\
\hline & $(1.22 \mathrm{~g})$ & $1.38(\mathrm{efg})$ & $(3.77 \mathrm{~d})$ & $(1.22 \mathrm{~h})$ & $(1.75 \mathrm{~g})$ & $(1.43 \mathrm{gh})$ & $(1.01 \mathrm{~h})$ & $(1.19 \mathrm{~h})$ & $(6.05 \mathrm{e})$ & $(1.84 \mathrm{j})$ & $(2.34 \mathrm{ij})$ & $(7.21 \mathrm{f})$ \\
\hline Penoxsulam $24 \%$ & 0.0 & 0.1 & 1.0 & 0.0 & 0.0 & 1.3 & 0.0 & 0.1 & 1.0 & 0.0 & 0.2 & 3.2 \\
\hline SC & $(0.71 \mathrm{~h})$ & $(0.73 \mathrm{~h})$ & $(1.32 \mathrm{fg})$ & $(0.71 \mathrm{i})$ & (0.79 i) & $(1.20 \mathrm{~h})$ & $(0.71 \mathrm{~h})$ & $(0.75 \mathrm{~h})$ & $(1.15 \mathrm{~h})$ & $(0.71 \mathrm{k})$ & $(0.85 \mathrm{k})$ & $(1.90 \mathrm{j})$ \\
\hline \multirow{2}{*}{ Hand weeding } & 1.7 & 6.8 & 6.7 & 2.2 & 2.6 & 15.9 & 4.2 & 4.8 & 39.3 & 8.0 & 14.2 & 61.9 \\
\hline & $1.62(\mathrm{efg})$ & (1.75 ef) & $(4.05 \mathrm{~d})$ & $(1.46 \mathrm{gh})$ & $(2.7 \mathrm{f})$ & $(2.68 \mathrm{f})$ & $(2.15 \mathrm{~g})$ & $(2.28 \mathrm{~g})$ & $(6.31 \mathrm{e})$ & $(2.92 \mathrm{i})$ & $(3.83 \mathrm{~h})$ & $(7.90 \mathrm{e})$ \\
\hline \multirow{2}{*}{ Weedy check } & 130.8 & 148.7 & 285.0 & 31.9 & 44.1 & 71.1 & 245.2 & 323.8 & 466.0 & 408.0 & 516.6 & 822.1 \\
\hline & $(5.70 \mathrm{c})$ & $(6.68 \mathrm{~b})$ & $(8.46 \mathrm{a})$ & $(11.46 \mathrm{c})$ & $(12.21 \mathrm{~b})$ & (16.90 a) & $(15.67 \mathrm{c})$ & $(18.00 \mathrm{~b})$ & $(21.59 \mathrm{a})$ & $(20.12 c)$ & $(22.74 \mathrm{~b})$ & $(28.68 \mathrm{a})$ \\
\hline
\end{tabular}

Means followed by a common letter within a season are not significantly different at $5 \%$ level, using Duncan s Multiple Range Test. DAT= days after herbicidal treatment 
Transplanting rice seedlings at 21 days old as well as 28 days old recorded the lowest dry weight of weed species and total weeds with herbicide treatment of penoxsulam $24 \% \mathrm{SC}$ at the rate of $0.029 \mathrm{~kg}$ ai $\mathrm{ha}^{-1}$ followed by oxadiazon $25 \% \mathrm{EC}$ at the rate of $0.045 \mathrm{~kg}$ ai ha ${ }^{-1}$ under the same two seedling ages (21 and 28 days) as compared to weedy check plots which gave the highest dry weight of weeds species and total weeds under 35 day seedling old. The same trend was observed during two season of study. The reason in reduction of dry weight of weeds due to fast rice plant growth and tillers production through early growth stage which caused suppress weeds and also, addition to weed management by herbicide application which reduced weed competition resulted in higher weed control under the younger seedling as compared to older seedlings as mentioned by Shukla et al. (2015) and Sinha et al. (2018).

b- Rice:

b.1. Effect of seedling ages and weed control treatments on dry weight $\left(\mathrm{gm}^{-2}\right)$, number of panicle $\mathrm{m}^{-2}$, panicle weight $(\mathrm{g})$, number of filled grain panicle $^{-1}, 1000$-grain weight $(\mathrm{g})$ and rice grain yield $\left(\mathrm{t} \mathrm{ha}^{-1}\right)$ during 2016 and 2017 seasons:

As shown in Tables (3 and 4), all measured traits were significantly affected by seedling ages. The highest dry weight, number of panicles $\mathrm{m}^{-2}$, panicle weight, number of filled grain panicle ${ }^{-1}$, 1000-grain weight and grain yield of rice were obtained when using the youngest seedling (21 days) in the first and second seasons.

Table (3): Dry weight $\left(\mathrm{g} \mathrm{m}^{-2}\right)$, number of panicles $\mathrm{m}^{-2}$ and panicle weight $(\mathrm{g})$ of rice as affected by seedling ages and weed control treatments in 2016 and 2017 seasons

\begin{tabular}{|c|c|c|c|c|c|c|}
\hline \multirow{2}{*}{$\begin{array}{l}\text { Treatments } \\
\text { a- Seedling age }\end{array}$} & \multicolumn{2}{|c|}{$\begin{array}{l}\text { Rice dry weight } \\
\qquad\left(\mathrm{g} \mathrm{m}^{-2}\right)\end{array}$} & \multicolumn{2}{|c|}{ Number of panicle $\mathrm{m}^{-2}$} & \multicolumn{2}{|c|}{$\begin{array}{c}\text { Panicle weight } \\
\text { (g) }\end{array}$} \\
\hline & \multicolumn{6}{|c|}{2016 Season } \\
\hline 21 days & $753.0 \mathrm{a}$ & $813.8 \mathrm{a}$ & $421.7 \mathrm{a}$ & $446.7 \mathrm{a}$ & $2.14 \mathrm{a}$ & $2.19 \mathrm{a}$ \\
\hline 28 day $s$ & $687.1 \mathrm{~b}$ & $746.1 \mathrm{~b}$ & $373.3 \mathrm{~b}$ & $400.0 \mathrm{~b}$ & $1.93 \mathrm{~b}$ & $2.06 \mathrm{~b}$ \\
\hline 35 day $s$ & $463.7 \mathrm{c}$ & $514.3 \mathrm{c}$ & $328.3 \mathrm{c}$ & $346.7 \mathrm{c}$ & $1.76 \mathrm{c}$ & $1.84 \mathrm{c}$ \\
\hline F. test & $* *$ & $* *$ & $* *$ & $* *$ & $* *$ & ** \\
\hline b- Weed control & \multicolumn{6}{|c|}{2017 Season } \\
\hline Thiobencarb $50 \%$ EC & $634.2 \mathrm{~d}$ & $675.6 \mathrm{~d}$ & $397.2 \mathrm{c}$ & $413.9 \mathrm{c}$ & $1.98 \mathrm{~b}$ & $2.04 \mathrm{~b}$ \\
\hline Oxadiazon $25 \%$ EC & $735.3 \mathrm{~b}$ & $795.4 \mathrm{~b}$ & $427.8 \mathrm{~b}$ & $450.0 \mathrm{~b}$ & $2.07 \mathrm{a}$ & $2.15 \mathrm{a}$ \\
\hline Penoxsulam $24 \%$ SC & $809.2 \mathrm{a}$ & $881.7 \mathrm{a}$ & $452.8 \mathrm{a}$ & $472.2 \mathrm{a}$ & $2.09 \mathrm{a}$ & $2.19 \mathrm{a}$ \\
\hline Hand weeding & $712.8 \mathrm{c}$ & $781.1 \mathrm{c}$ & $400.0 \mathrm{c}$ & $425.0 \mathrm{c}$ & $1.98 \mathrm{~b}$ & $2.06 \mathrm{~b}$ \\
\hline Weedy check & $281.4 \mathrm{e}$ & $323.2 \mathrm{e}$ & $194.4 \mathrm{~d}$ & $227.8 \mathrm{~d}$ & $1.62 \mathrm{c}$ & $1.71 \mathrm{c}$ \\
\hline F. test & $* *$ & $* *$ & $* *$ & $* *$ & $* *$ & $* *$ \\
\hline Interaction $(\mathrm{a} * \mathrm{~b})$ & $* *$ & $* *$ & $* *$ & $* *$ & $* * * *$ & $* *$ \\
\hline
\end{tabular}


On the other hand, the oldest seedling age (35 days) gave the lowest values of these traits. The increase in these above mentioned characters might be attributed to the ability of younger seedlings to greater root development of rice plant and activity, high ability to use better utilization of growth resources from soil more than oldest seedlings (Singh et al., 2013) and produced more tillers due to quick regeneration of seedlings and plant vigor (Haque, 2002), increase ability of young seedlings at early transplanting stage on produced more number of leaves resulting in higher photosynthesis and producing more dry matter (Damodaran et al., 2012), in addition to, increase number of panicles and panicle weight which consequently improved the grain yield (Ramachandra et al., 2012).

As for weed control treatments, it is clear from the results in Tables ( 3 and 4) all weed control treatments significantly increased dry weight, number of panicle $\mathrm{m}^{-2}$, panicle weight, number of filled grains panicle $^{-1}, 1000$-grain weight and grain yield of rice than untreated check plots in 2016 and 2017 seasons. Penoxsulam 24\% SC at the rate of $0.029 \mathrm{~kg}$ ai ha ${ }^{-1}$ recorded the greatest of the studied characters as well as oxadiazon $25 \% \mathrm{EC}$ at the rate of at $0.045 \mathrm{~kg}^{\mathrm{ai}} \mathrm{ha}^{-1}$ on panicle weight as compared to weedy check plots, which gave the lowest values ones in the first and second seasons without significant differences between oxadiazon $25 \%$ EC at the rate of at $0.045 \mathrm{~kg}$ ai ha ${ }^{-1}$ on 1000-grain weight in the first season. This result might be due to effective chemical control of weeds during early growth stages of rice, resulted in lower competition with rice plants on moisture, nutrients and sunlight which in turn significantly increased dry weight, number of panicles $\mathrm{m}^{-2}$ and panicle weight, that resulted in increase rice grain yield as found by Singh et al. (2005) and Sakar et al. (2017).

Table (4): Number of filled grains panicle $\mathrm{e}^{-1}, 1000$-grain weight $(\mathrm{g})$ and grain yield $\left(\mathrm{t}^{-1}\right)$ of rice as affected by seedling ages and weed control treatments in 2016 and 2017 seasons

\begin{tabular}{|c|c|c|c|c|c|c|}
\hline \multirow{2}{*}{$\begin{array}{l}\text { Treatments } \\
\text { a- Seedling age: }\end{array}$} & \multicolumn{2}{|c|}{$\begin{array}{l}\text { Number of filled grains } \\
\text { panicle }^{-1}\end{array}$} & \multicolumn{2}{|c|}{$\begin{array}{l}\text { 1000-grain weight } \\
\text { (g) }\end{array}$} & \multicolumn{2}{|c|}{$\begin{array}{l}\text { Grain yield } \\
\qquad\left(\mathrm{t} \mathrm{ha}^{-1}\right)\end{array}$} \\
\hline & 2016 & 2017 & 2016 & 2017 & 2016 & 2017 \\
\hline 21 days & $99.7 \mathrm{a}$ & $102.9 \mathrm{a}$ & $23.3 \mathrm{a}$ & $24.1 \mathrm{a}$ & $7.9 \mathrm{a}$ & $8.2 \mathrm{a}$ \\
\hline 28 days & $94.4 \mathrm{~b}$ & $97.1 \mathrm{~b}$ & $22.3 \mathrm{~b}$ & $23.2 \mathrm{~b}$ & $7.0 \mathrm{~b}$ & $7.4 \mathrm{~b}$ \\
\hline 35 days & $85.3 \mathrm{c}$ & $88.5 \mathrm{c}$ & $21.3 \mathrm{c}$ & $21.8 \mathrm{c}$ & $5.7 \mathrm{c}$ & $6.1 \mathrm{c}$ \\
\hline F. test & $* *$ & $* *$ & $* *$ & $* *$ & $* *$ & $* *$ \\
\hline
\end{tabular}

b- Weed control:

\begin{tabular}{lcccccc}
\hline Thiobencarb 50\% EC & $94.3 \mathrm{~d}$ & $96.7 \mathrm{~d}$ & $23.3 \mathrm{c}$ & $24.0 \mathrm{~b}$ & $7.4 \mathrm{~d}$ & $7.8 \mathrm{c}$ \\
Oxadiazon 25 \% EC & $99.3 \mathrm{~b}$ & $102.9 \mathrm{~b}$ & $23.7 \mathrm{ab}$ & $24.3 \mathrm{~b}$ & $8.0 \mathrm{~b}$ & $8.5 \mathrm{~b}$ \\
Penoxsula 24\% SC & $105.0 \mathrm{a}$ & $107.7 \mathrm{a}$ & $23.9 \mathrm{a}$ & $24.6 \mathrm{a}$ & $8.5 \mathrm{a}$ & $8.8 \mathrm{a}$ \\
Hand weeding & $96.1 \mathrm{c}$ & $99.4 \mathrm{c}$ & $23.4 \mathrm{bc}$ & $23.9 \mathrm{~b}$ & $7.6 \mathrm{c}$ & $7.9 \mathrm{c}$ \\
Weedy check & $70.9 \mathrm{e}$ & $74.2 \mathrm{e}$ & $17.0 \mathrm{~d}$ & $18.1 \mathrm{c}$ & $2.8 \mathrm{e}$ & $3.1 \mathrm{~d}$ \\
\hline F. test & $* *$ & $* *$ & $* *$ & $* *$ & $* *$ & $* *$ \\
\hline Interaction $(\mathrm{A} * \mathrm{~B})$ & $* *$ & $* *$ & $* *$ & $* *$ & $* *$ & $* *$
\end{tabular}

** indicate $\mathrm{P}<0.05$ and $\mathrm{P}<0.01$. Means of transformed data followed by the same letter are not significantly different at $5 \%$ level, using Duncan's Multiple Range Test 
b.2. Effect of interaction between seedling ages and weed control treatments on dry weight $\left(\mathrm{g} \mathrm{m}^{-2}\right)$, number of panicles $\mathrm{m}^{-2}$, panicle weight $(\mathrm{g})$, number of filled grain panicle ${ }^{-1}, 1000$-grain weight $(\mathrm{g})$ and grain yield $\left(\mathrm{t} \mathrm{h}^{-1}\right)$ of rice during 2016 and 2017 seasons:

As shown in Tables (5 and 6), dry weight, number of panicle $\mathrm{m}^{-2}$, panicle weight, number of filled grains panicle $^{-1}, 1000$-grain weight and grain yield of rice were significantly influenced by the interaction between seedling ages and weed control treatment during the two seasons.

The highest dry weight, number of panicles $\mathrm{m}^{-2}$, panicle weight, number of filled grains panicle ${ }^{-1}, 1000-$ grain weight and grain yield of rice were recorded by the combination of planted rice at 21 days old seedling when treated by penoxsulam $24 \% \mathrm{SC}$ at the rate of $0.029 \mathrm{~kg}$ ai ha ${ }^{-1}$ as well as oxadiazon $25 \% \mathrm{EC}$ at the rate of at $0.045 \mathrm{~kg}$ ai ha ${ }^{-1}$ on panicle weight without significant differences between oxadiazon $25 \%$ EC on 1000 -grain weight during two seasons of study. On the other side, the lowest values of these characters were observed in the interaction between transplanted rice at 35 days old seedling under untreated plots (weedy check) during two seasons of study. The increase of rice characters might be due to increase plant density, resulting in higher photosynthesis (Rasool et al., 2012). In addition, due to increasing in number of panicles $\mathrm{m}^{-}$ ${ }^{2}$, panicle weight and 1000-grain weight, resulting in increased rice grain yield (Thiyagarajan et al., 2002 and Ramachandra et al., 2012) moreover, weed suppression by herbicide application, which effectively reduced weed competition, significantly increased number of panicles $\mathrm{m}^{-2}$ and panicle weight consequently improved the grain yield as mentioned by Singh et al. (2005), Ramachandra et al. (2012) and Shukla et al. (2014).

Table (5): Dry weight $\left(\mathrm{g} \mathrm{m}^{-2}\right)$, number of panicles $\mathrm{m}^{-2}$ and panicle weight $(\mathrm{g})$ of rice as affected by the interaction between seedling ages and weed control treatments in 2016 and 2017 seasons

\begin{tabular}{|c|c|c|c|c|c|c|c|c|c|}
\hline \multirow{4}{*}{$\begin{array}{l}\text { Weed control } \\
\text { treatments }\end{array}$} & \multicolumn{3}{|c|}{ Dry weight $\left(\mathrm{g} \mathrm{m}^{-2}\right)$} & \multicolumn{3}{|c|}{ Number of panicles $\mathrm{m}^{-2}$} & \multicolumn{3}{|c|}{ Panicle weight (g) } \\
\hline & \multicolumn{9}{|c|}{ Seedling age } \\
\hline & 21 days & 28 days & 35 days & 21 days & 28 days & 35 days & 21 days & 28 days & 35 days \\
\hline & \multicolumn{9}{|c|}{2016 Season } \\
\hline $\begin{array}{l}\text { Thiobencarb } \\
50 \% \text { EC }\end{array}$ & $734.3 \mathrm{~d}$ & $661.3 \mathrm{e}$ & $507.0 \mathrm{~g}$ & $433.3 \mathrm{c}$ & $391.7 \mathrm{de}$ & 366.7 ef & $2.15 \mathrm{bc}$ & 1.94 ef & $1.83 \mathrm{gh}$ \\
\hline $\begin{array}{l}\text { Oxadiazon } 25 \% \\
\text { EC }\end{array}$ & $856.0 \mathrm{~b}$ & $807.9 \mathrm{c}$ & $541.9 \mathrm{fg}$ & $475.0 \mathrm{~b}$ & $433.3 \mathrm{c}$ & $375.0 \mathrm{def}$ & $2.33 \mathrm{a}$ & $2.01 \mathrm{de}$ & $1.86 \mathrm{fgh}$ \\
\hline $\begin{array}{l}\text { Penoxsulam } \\
24 \% \text { SC }\end{array}$ & 971.9 a & $880.7 \mathrm{~b}$ & $575.0 \mathrm{f}$ & $525.0 \mathrm{a}$ & $450.0 \mathrm{bc}$ & $383.3 \mathrm{def}$ & $2.30 \mathrm{a}$ & $2.07 \mathrm{~cd}$ & $1.91 \mathrm{efg}$ \\
\hline Hand weeding & $814.2 \mathrm{c}$ & $807.1 \mathrm{c}$ & $517.1 \mathrm{~g}$ & $441.7 \mathrm{c}$ & $400.0 \mathrm{~d}$ & $358.3 \mathrm{f}$ & $2.17 \mathrm{~b}$ & $1.96 \mathrm{ef}$ & $1.81 \mathrm{gh}$ \\
\hline \multirow[t]{2}{*}{ Weedy check } & $388.7 \mathrm{~h}$ & $278.2 \mathrm{i}$ & $177.3 \mathrm{j}$ & $233.3 \mathrm{~g}$ & $191.7 \mathrm{~h}$ & $158.3 \mathrm{i}$ & $1.76 \mathrm{hi}$ & $1.68 \mathrm{i}$ & $1.40 \mathrm{j}$ \\
\hline & \multicolumn{9}{|c|}{2017 Season } \\
\hline $\begin{array}{l}\text { Thiobencarb } \\
50 \% \text { EC }\end{array}$ & 778.7 e & $687.3 \mathrm{f}$ & $560.9 \mathrm{i}$ & $450.0 \mathrm{~cd}$ & $408.3 \mathrm{ef}$ & $383.3 \mathrm{f}$ & $2.22 \mathrm{bc}$ & $2.07 \mathrm{~d}$ & $1.85 \mathrm{ef}$ \\
\hline $\begin{array}{l}\text { Oxadiazon } 25 \% \\
\text { EC }\end{array}$ & $896.7 \mathrm{c}$ & $900.0 \mathrm{c}$ & $589.5 \mathrm{~g}$ & $500.0 \mathrm{~b}$ & $450.0 \mathrm{~cd}$ & $400.0 \mathrm{ef}$ & $2.37 \mathrm{a}$ & $2.17 \mathrm{bcd}$ & $1.91 \mathrm{e}$ \\
\hline $\begin{array}{l}\text { Penoxsulam } \\
24 \% \text { SC }\end{array}$ & $1104.0 \mathrm{a}$ & $935.6 \mathrm{~b}$ & $605.6 \mathrm{~g}$ & $541.7 \mathrm{a}$ & $475.0 \mathrm{bc}$ & $400.0 \mathrm{ef}$ & $2.42 \mathrm{a}$ & $2.23 \mathrm{~b}$ & $1.92 \mathrm{e}$ \\
\hline Hand weeding & $870.6 \mathrm{~d}$ & $892.4 \mathrm{~cd}$ & $580.2 \mathrm{gh}$ & $466.7 \mathrm{c}$ & $425.0 \mathrm{de}$ & $383.3 \mathrm{f}$ & $2.18 \mathrm{bcd}$ & $2.11 \mathrm{~cd}$ & $1.88 \mathrm{ef}$ \\
\hline Weedy check & $418.8 \mathrm{i}$ & $315.4 \mathrm{j}$ & $235.3 \mathrm{k}$ & $275.0 \mathrm{~g}$ & $241.7 \mathrm{~h}$ & $166.7 \mathrm{i}$ & $1.78 \mathrm{fg}$ & $1.70 \mathrm{gh}$ & $1.63 \mathrm{~h}$ \\
\hline
\end{tabular}

Means followed by a common letter within a season are not significantly different at 5\% level, using Duncans Multiple Range Test. DAT $=$ days after herbicidal treatment 
Table (6): Number of filled grains panicles ${ }^{-1}, 1000$-grain weight $(\mathrm{g})$ and grain yield $\left(\mathrm{t} \mathrm{ha} \mathrm{h}^{-1}\right)$ of rice as affected by the interaction between seedling age and weed control treatments in 2016 and 2017 seasons

\begin{tabular}{|c|c|c|c|c|c|c|c|c|c|}
\hline \multirow{4}{*}{$\begin{array}{l}\text { Weed control } \\
\text { treatments }\end{array}$} & \multicolumn{3}{|c|}{$\begin{array}{l}\text { Number of filled grain } \\
\text { panicle }^{-1}\end{array}$} & \multirow{2}{*}{\multicolumn{3}{|c|}{$\begin{array}{c}\begin{array}{c}\text { 1000-grain weight } \\
(\mathrm{g})\end{array} \\
\text { Seedling age }\end{array}$}} & \multicolumn{3}{|c|}{$\begin{array}{l}\text { Grain yield } \\
\left(\mathrm{t} \mathrm{ha}^{-1}\right)\end{array}$} \\
\hline & & & & & & & & & \\
\hline & 21 days & 28 days & $\begin{array}{c}35 \\
\text { days }\end{array}$ & 21 days & 28 days & 35 days & $\begin{array}{c}21 \\
\text { days }\end{array}$ & $\begin{array}{c}28 \\
\text { days }\end{array}$ & $\begin{array}{c}35 \\
\text { days }\end{array}$ \\
\hline & \multicolumn{9}{|c|}{2016 Season } \\
\hline $\begin{array}{l}\text { Thiobencarb } 50 \% \\
\text { EC }\end{array}$ & $100.0 \mathrm{~cd}$ & 95.7 ef & $87.3 \mathrm{~h}$ & $23.98 \mathrm{bc}$ & $23.17 \mathrm{def}$ & $22.57 \mathrm{f}$ & $8.18 \mathrm{~d}$ & $7.47 \mathrm{e}$ & $6.46 \mathrm{~h}$ \\
\hline $\begin{array}{l}\text { Oxadiazon } 25 \% \\
\text { EC }\end{array}$ & $109.0 \mathrm{~b}$ & $98.7 \mathrm{~d}$ & $90.3 \mathrm{~g}$ & $24.44 \mathrm{ab}$ & $23.77 \mathrm{bcd}$ & $22.87 \mathrm{ef}$ & $9.16 \mathrm{~b}$ & $8.12 \mathrm{~d}$ & $6.81 \mathrm{~g}$ \\
\hline $\begin{array}{l}\text { Penoxsulam } 24 \% \\
\text { SC }\end{array}$ & $113.0 \mathrm{a}$ & $107.7 \mathrm{~b}$ & $94.3 \mathrm{f}$ & $25.05 \mathrm{a}$ & $23.93 \mathrm{bc}$ & $23.00 \mathrm{ef}$ & $9.45 \mathrm{a}$ & $8.96 \mathrm{bc}$ & $7.12 \mathrm{f}$ \\
\hline Hand weeding & $101.7 \mathrm{c}$ & $97.3 \mathrm{de}$ & $89.3 \mathrm{gh}$ & $24.13 \mathrm{bc}$ & 23.54 cde & $22.63 f$ & $8.78 \mathrm{c}$ & $7.70 \mathrm{e}$ & $6.22 \mathrm{~h}$ \\
\hline Weedy check & $75.0 \mathrm{i}$ & $72.7 \mathrm{i}$ & $65.0 \mathrm{j}$ & $18.77 \mathrm{~g}$ & $16.90 \mathrm{~h}$ & $15.43 \mathrm{i}$ & $3.96 \mathrm{i}$ & $2.68 \mathrm{j}$ & $1.71 \mathrm{k}$ \\
\hline \multicolumn{10}{|c|}{2017 Season } \\
\hline $\begin{array}{l}\text { Thiobencarb } 50 \% \\
\text { EC }\end{array}$ & $101.3 \mathrm{e}$ & $98.7 \mathrm{fg}$ & $90.0 \mathrm{i}$ & $24.73 \mathrm{bc}$ & $24.03 \mathrm{~d}$ & $23.33 \mathrm{e}$ & $8.50 \mathrm{e}$ & $7.90 \mathrm{f}$ & $6.92 \mathrm{~h}$ \\
\hline $\begin{array}{l}\text { Oxadiazon } 25 \% \\
\text { EC }\end{array}$ & $114.0 \mathrm{~b}$ & $101.3 \mathrm{e}$ & $93.3 \mathrm{~h}$ & $25.23 \mathrm{ab}$ & $24.57 \mathrm{~cd}$ & $22 . .97 \mathrm{e}$ & $9.45 \mathrm{~b}$ & $8.77 \mathrm{de}$ & $7.26 \mathrm{~g}$ \\
\hline $\begin{array}{l}\text { Penoxsulam } 24 \% \\
\text { SC }\end{array}$ & $116.7 \mathrm{a}$ & $109.3 \mathrm{c}$ & $97.0 \mathrm{~g}$ & $25.50 \mathrm{a}$ & $24.90 \mathrm{bc}$ & $23.33 \mathrm{e}$ & $9.78 \mathrm{a}$ & $9.10 \mathrm{c}$ & $7.53 \mathrm{~g}$ \\
\hline Hand weeding & $104.7 \mathrm{~d}$ & 100.7 ef & $91.3 \mathrm{hi}$ & $24.77 \mathrm{bc}$ & $24.33 \mathrm{~cd}$ & $22.87 \mathrm{e}$ & $9.03 \mathrm{~cd}$ & $7.96 \mathrm{f}$ & $6.79 \mathrm{~h}$ \\
\hline Weedy check & $78.0 \mathrm{j}$ & $75.7 \mathrm{k}$ & 69.01 & $20.03 \mathrm{f}$ & $17.97 \mathrm{~g}$ & $16.30 \mathrm{~h}$ & $4.26 \mathrm{i}$ & $3.11 \mathrm{j}$ & $1.95 \mathrm{k}$ \\
\hline
\end{tabular}

\section{CONCLUSIONS}

Based on the obtained results of this study it could be concluded that, the highest rice grain yield and best weed control were achieved by transplanting rice seedlings at 21 days old and also weed control treatment by using penoxsulam $24 \% \mathrm{SC}$ at the rate of $0.029 \mathrm{~kg}$ ai ha ${ }^{-1}$ (Granite 24\% SC) applied at 15 DAT.

\section{REFERENCES}

Abou-Khalifa, A. A. (2005). Physiological behavior of same rice cultivars under different sowing dates and seedling ages. The 11th Conf. Agron. Dept., Fac., on Agric., Assiut Univ., Nov., 15-16: 314-323.

Ahmad, M., T. H. Awan, G. M. Sarwar, M. Ahmad and S. Yaseen (2004). Harmful insects, diseases, weeds of rice and their control. pp.7

Anitha, S. and M. Chellappan (2011). Comparison of the SRI recommended practices, and farmers' methods of rice production in the humid tropics of Kerala, India. Journal of Tropical Agriculture, 49 (1-2): 64-71.
Chauhan, B. S. (2012). Weed ecology and weed management strategies for dry-seeded rice in Asia. Weed Technol., 26, 1-13.

Damodaran, V., B. K. Saren, N. Ravisankar and N. Bommayasamy (2012). Influence of time of planting, spacing, seedling number and nitrogen management practices on productivity, profitability and energetic of rice in Island ecosystem. Madras Agricultural Journal, 99(7-9): 538-544.

Drost, D. C. and K. Moody (1982). Effect of butachlor on Echinichloa glubrescensin wet seeded rice (Oryza sativa L.) Philippines J. Weed Sci., 9:44-57.

Duncan, D. B. (1955). Multiple range and multiple Ftests. Biometrics 11: 1-42.

El-Kassaby, A. T., M. H. Ghonima, A. A. Abd-Allah and T. M. El-Hefnawy (2012). Effect of seedling age and plant spacing on growth characters and yield of some rice cultivars. J. Plant Production, Mansoura Univ., 3(4): 705 714.

El-Khoby. M. W. and Alaa M. E. A. Shahein (2016). Effect of irrigation management and seedling 
age on the productivity and quality of two rice varieties using system of rice intensification (SRI) J. Agric. Res. Kafr El-Sheikh Univ., 42(3): 288-299.

FAOSTAT (2017). FAO statistical database. Available at faostat. External. FAO. Org./faostat.

Ginigaddara, G. A. S. and S. L. Ranamukhaarachchi (2011). Study of age of seedling at transplanting on growth dynamics and yield of rice under alternating flooding and suspension of irrigation of water management. Recent Research in Science and Technology, 3(3): 76-88.

Haque, D. E. (2002). Effect of Madagascar Technique of younger seedling and wider spacing on growth and yield of Boro rice. M.S. Thesis, Dept. Agron., Bangladesh Agril. Univ. Mymensingh, pp. 28-71.

Hassan, S. M. (1999). Weed management in rice. Advances in Agric. Res. in Egypt (Special Issue) 2(3):220-247.

Karim, S. M. R., T. M. T. Iqbal and N. Islam. (1998). Relative yields of crops and crop losses due to weed competition in Bangladesh, Pak. J. Sci. Ind. Res., 41: 318-324.

Kavitha, M. P., V. Ganesaraja, V. K. Paulpandi and R. B. Subramanian (2010). Effect of age of seedlings, weed management practices and humic acid application on system of rice intensification. Indian J. Agric. Res., 44(4):294-299.

Kewat, M. L., S. B. Agrawal, K. K. Agrawal and R. S. Sharma (2002). Effect of divergent plant spacing and age of seedlings on yield and economics of hybrid rice. Indian J. Agron., 47 (3) : 367-371.

Krishna, A., N. K. Biradapatil, K. Manjappa and B. B. Channappagoudar (2008). Evaluation of system of rice intensification cultivation, seedling age and spacing on seed yield and quality in "Samba Mahsuri" (BPT-5204) rice, Karnataka Journal of Agricultural Sciences, 21(1):20-25.

Kumar, V. and J. K. Ladha (2011). Direct-seeding of rice: recent development and future research needs. Adv. Agron., 111, 297-413.

Labrada, R. (2001). FAO Training on Weed Management. P. 1-8. In: Proc. of the $18^{\text {th }}$ Asian-Pacific Weed Sci. Soc., Conf. May 28 - June 2, Beijing, China.

Mondal, M. M. A., A. B. Putech, M. R. Ismail and M. Y. Rafii (2013). Optimizing plant spacing for modern rice varieties. International J., of Agric. \& Biol., 15(1):175-178.

Montazeri, M. P. A., R. Hemadi, A. D. Rivand and M. Jabbari (2010). Evaluation on the efficacy of herbicide Penoxsulam $240 \mathrm{SC}$ in rice fields. AGRIS-FAO.

Paramita, B., D. Dutta, S. Biswas, P. Bandyoopadhay and D. Maiti (2005). Effect of irrigation and weed management on weed growth and yield performance of transplanted hybrid rice. Journal of Crop and weed, 2(1): 20-22.
Rahman, M., J. Abdul Shukor, A. S. M. Jaya Suria, A. B. Man and P. Anwar (2012). Response of weed flora to different herbicides in aerobic rice system. Scientific Research and Essays, 7(1): $12-23$.

Ramachandra, C., N. Shivakumar, M. P. Rajanna and K. N. Kalyanamurthy (2012). Effect of age of seedlings and weed management under SRI on yield of rice. Indian Journal of Weed Science, 44(1): 50-52.

Ranjit, J. D. (1997). Weeds and weed management in rice-wheat system. In: Proceedings of the Rice-Wheat Research. End of Project Workshop held at Kathmandu, Nepal from Oct. 1-3, pp. 23-30. [C.F. CD Rom Computer System].

Rasool, F. Ur., R. Habib and M. I. Bhat (2012). Evaluation of plant spacing and seedlings hill1 on rice (Oryza Sativa L.) productivity under temperate conditions. Pakisthan Journal of Agricultural Science, 49(2): 169-172.

Rekha, K. B., M. S. Raju and M. D. Reddy (2003). Effect of herbicides on weed growth, grain yield and nutrient uptake in rain fed low land rice. : Indian, J. Weed Sci., 35: 121-122.

Roy, A. (2015). Effect of age of seedlings at staggered planting and nutrient management on the growth and yield of aromatic fine rice (cv. BRRI dhan 38) M.S. Thesis, Dept. Agron. Bangladesh Agril. Univ., Mymensingh. p 2.

Salem, A. K. M., W. M. ElKhoby, A. B. Abou-Khalifa and M. Ceesay (2011). Effect of nitrogen fertilizer and seedling age on inbred and hybrid rice varieties. American-Eurasian J. Agric. and Environ. Sci., 11 (5): 640-646.

Sandeep, N, S., K. S. Singh, R. K. Panwar, S. Malik; S. Narwal and S. Singh (2002). Performance of acetachlor and anilofos+ethoxysulfuron for weed control in transplanted rice (Oryza sativa). Indian J. Agron., 47 (1): 67-71.

Sarkar, M. A. R., S. K. Paul and U. Paul (2017). Effect of water and weed management in Boro rice (cv. BRRI dhan28) in Bangladesh. Archives of Agriculture and Environmental Science, 2(4): 325-329.

Shebl, S. M., I. H. Abou El-Darag and H. F. EL-Mwafi (2009). Effect of varietal performance and weed control efficacy on weeds, growth and yield of hybrid rice. J. Agric. Res. Kafr ElSheikh Univ., 35(1): 127-148.

Shukla, U. N., V. K. Srivastava, S. Singh, A. Sen and V. Kumar (2014). Growth, yield and economic potential of rice (Oryza sativa) as influenced by different age of seedlings, cultivars and weed management under system of rice intensification. Indian Journal of Agricultural Sciences, 84(5): 628-36.

Shukla, U. N., V. K. Srivastava, S. Smita, U. S. Ram and A. K. Pandey (2015). Effect of seedlings age, cultivars and weed management on weed dynamics, nutrient removal and yield of rice (Oryza sativa L.) under system of rice 
intensification (SRI). Indian Journal of Agricultural Sciences, 85(10): 1260-8.

Singh, K., S. R. Singh, J. K. Singh, R. S. Rathore, S. Pal, S. P. Singh and R. Roy (2013). Effect of age of seedling and spacing on yield, economics, soil health and digestibility of rice (Oryza sativa) genotypes under system of rice intensification. Indian Journal of Agricultural Sciences, 83(5): 479-83.

Singh, V. P., S. Govindra, R. K. Singh, S. P. Singh, A. Kumar, V.C. Dhyani, M. Kumar and G. Sharma (2005). Effect of herbicides alone and in combination on direct-seeded rice. Indian Journal of Weed Science, 37: 197-201.

Singh, V. V., M. L. Jat, Z. A. Ganie, B. S. Chauhan and R. K. Gupta (2016). Herbicide options for effective weed management in dry directseeded rice under scented rice-wheat rotation of western Indo-Gangetic Plains. Crop Protection, 81: 168-176.

Sinha, T., S. K. Paul and Md. Abdur Rahman Sarkar (2018). Effect of age of seedlings at staggered transplanting and weed management on the growth and yield of aromatic Boro rice (cv. BRRI dhan50). J Bangladesh Agril. Univ., 16(1): 5-11.

Snedecor, G. W. and W. G. Cochran. (1971). Statistical Methods. $6^{\text {th }}$ ed. Iowa State Univ. Press Amer, USA.

Thakur, K. S. and K. Bassi. (1994). Efficacy of promising herbicides in direct-sown rainfed rice. Indian J. Weed Sci., 26(1\&2):75-78.

Thiyagarajan, T. M., K. Senthilkumar, P. S. Bindraban, H. Hengsdijk and S. Ramaswamy 2002. Crop management options for increasing water productivity in rice. Journal of Agricultural Resource Management, 1: 169-81

Upadhyay, V. B., R. Mathew, S. K. Vishwakarma and V. K. shukla (2003). Effect of number of seedlings per hill and age of seedlings on productivity and economics of transplanted rice. JNKVV Research Journal, 37(1): 27 29. 5 ref.

\section{تأثثير عمر الثتلات ومعاملات مكافحه الحثنائش علي نمو ومحصول الأرز الثتّل (سخا 7 ـ 1 )

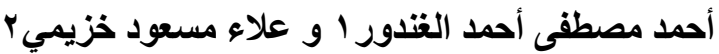

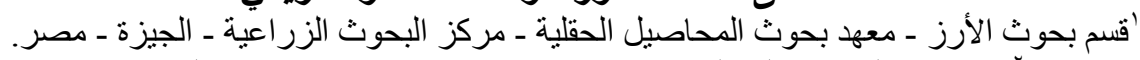 \\ كَسم وقاية النبات ـ كلية الزر اعة ـ جامعة دمنهور - جمهورية مصر العر العربية الجرية}

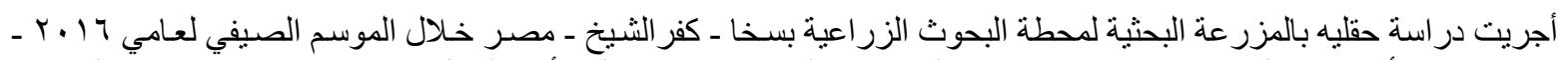

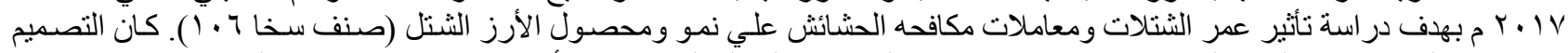

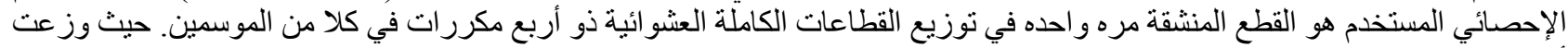

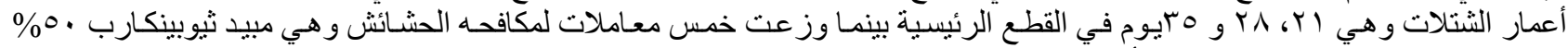

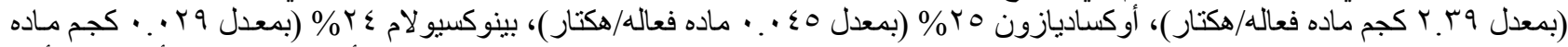

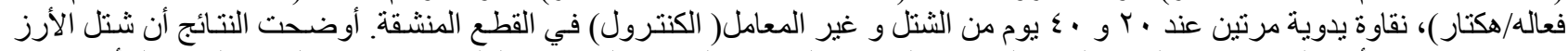

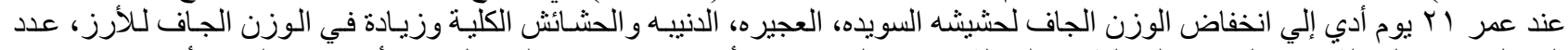

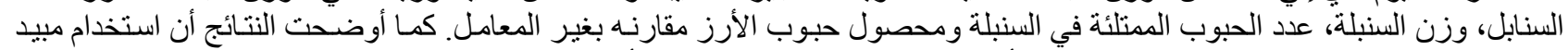

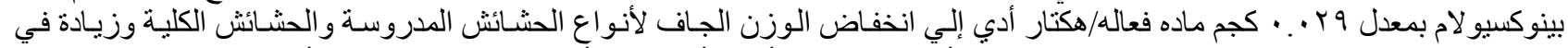

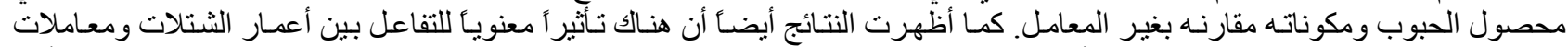

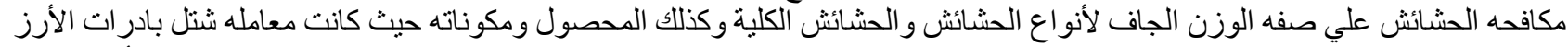

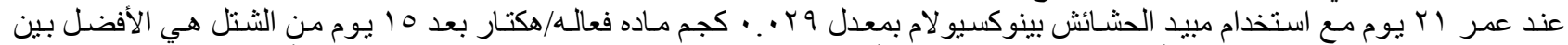

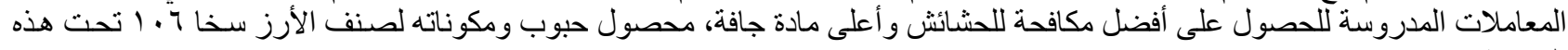

